\title{
Limits and Signatures of Relativistic Spaceflight
}

\author{
Ulvi Yurtsever ${ }^{*}$ and Steven Wilkinson ${ }^{\dagger}$ \\ Raytheon Company, El Segundo, CA 92195
}

(Dated: April 21, 2015)

\begin{abstract}
While special relativity imposes an absolute speed limit at the speed of light, our Universe is not empty Minkowski spacetime. The constituents that fill the interstellar/intergalactic vacuum, including the cosmic microwave background photons, impose a lower speed limit on any object travelling at relativistic velocities. Scattering of cosmic microwave phtotons from an ultra-relativistic object may create radiation with a characteristic signature allowing the detection of such objects at large distances.
\end{abstract}

\section{Introduction}

At a fixed speed $v$, the distance travelled by a spacecraft within a given (proper) travel time $\tau$ scales as $\gamma v \tau$, which for relativistic speeds approximately equals $\gamma c \tau$, where $\gamma=\left(1-v^{2} / c^{2}\right)^{-1 / 2}$. Hence range is directly proportional to $\gamma$, and to cover intergalactic distances within a limited "lifetime" $\tau$ requires $\gamma \gg 1$. The primary obstacles to relativistic $(\gamma \gg 1)$ space travel would be collisions with interstellar dust particles (cosmic dust) and larger space objects, which will impact with kinetic energies of $(\gamma-1) m c^{2}$ for rest mass $m$, similar to collisions in particle accelerators but potentially at much higher energies. For large enough $\gamma$, even molecular collisions could be a significant source of drag and possibly damaging. For example, at $\gamma=2$ a baseball size object of mass $150 \mathrm{~g}$ has an impact energy equivalent to 36 Megatons of TNT; a single cosmic dust grain of mass $10^{-14} \mathrm{~g}$ at $\gamma=10^{8}$ has an impact energy of close to $24 \mathrm{kgs}$ of TNT. [1-4]

impact energy in kgs of TNT equivalent

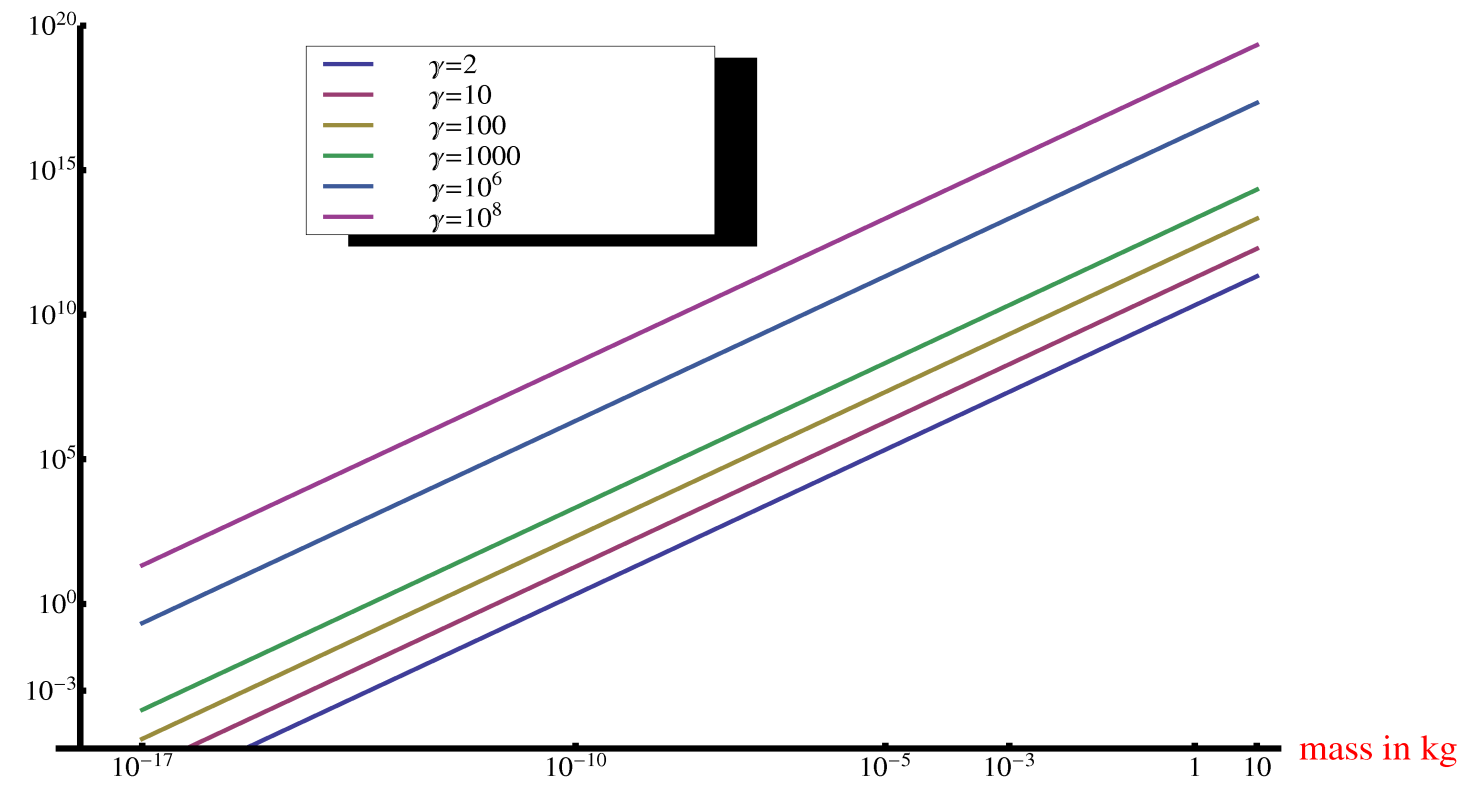

*Electronic address: ulvi.yurtsever@raytheon.com

†Electronic address: srwilkinson@raytheon.com 
distance travelled in lightyears

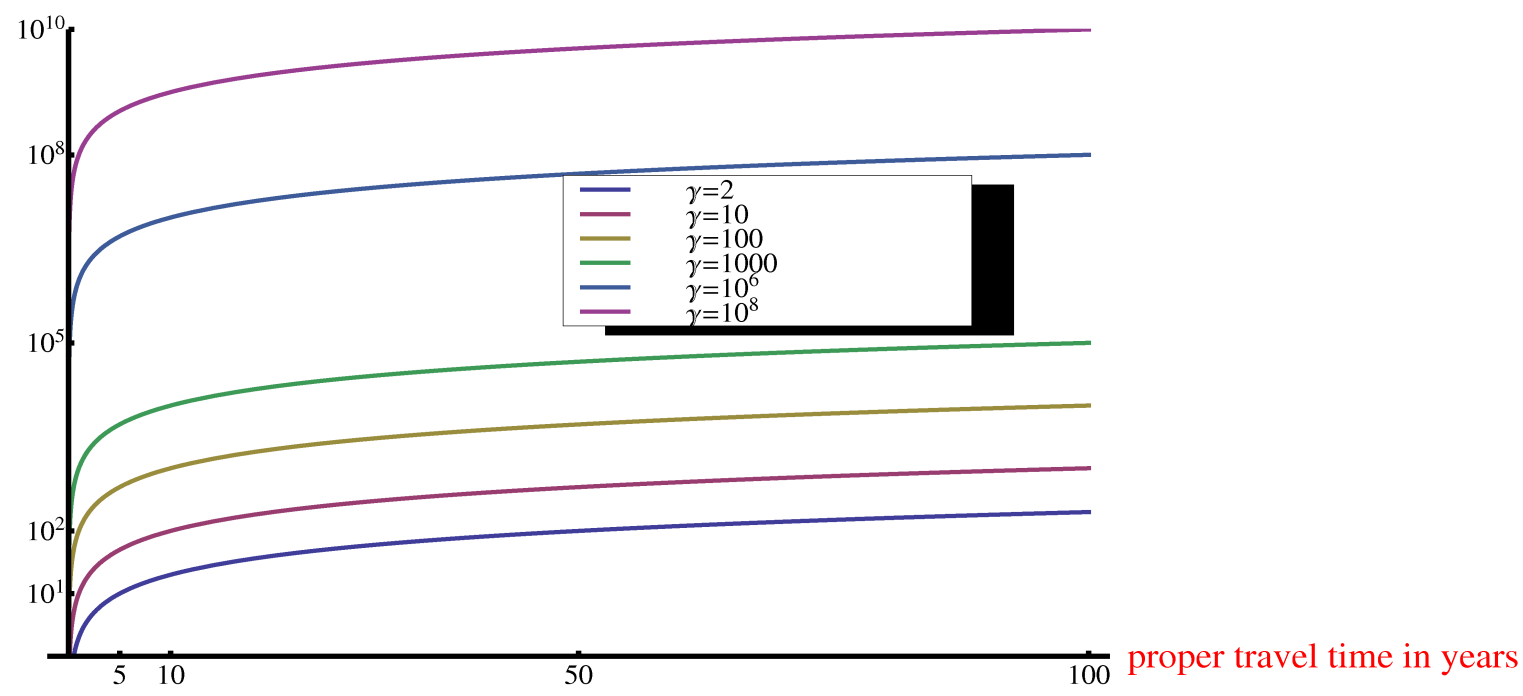

The intergalactic medium has much less debris per unit volume as compared to the interstellar medium so the chance of a catastrophic collision occurring is significantly reduced beyond the confines of any galaxy. We define extreme velocities to occur at $\gamma>9.1$ which is the threshold velocity for proton-proton collisions to produce antiprotons. Now to travel at a safe speed in the interstellar medium one would need a $\gamma<1.3$, the threshold for the pion production in proton-proton collisions. The problem of colliding with larger objects as described above remains, but we assume that sufficiently advanced technology likely to accompany relativistic space travel capabilitites will be able to circumvent these interactions. One can imagine, though, intergalactic travel through mostly matter-free space, and under those conditions the cosmic microwave background (CMB) photons are primarily the particles a spacecraft will encounter in collisions.

\section{CMB Interactions}

A fast-moving spacecraft traveling in intergalactic space still has to contend with collisions with cosmic microwave photons, which, at relativistic speeds, will appear in the spacecraft frame as highly energetic gamma rays. Interactions of $\mathrm{CMB}$ photons with the material of the spacecraft hull will have effects ranging from ionization and Compton scattering to pair production with increasing $\gamma$. We will assume that advanced technology can mitigate the harmful effets of ionization and Compton scattering, and therefore concentrate on pair production as the obstacle that is most likely to resist removal via technology. [1]

The first speed level where pair production will pose a challenge for relativistic spaceflight engineers is photonnucleon interactions in the hull of the spacecraft. Let us consider a hull made of ordinary baryonic matter. In this case, a single $\mathrm{CMB}$ photon will create an electron-positron pair via its collision with a nucleus in the hull if its energy exceeds the rest mass of an electron and positron. That threshold is at blueshifted photon energies of about $1 \mathrm{MeV}$. In terms of the frequency $\omega$ in the rest frame of the spacecraft, the condition for nucleus-mediated pair production is

$$
\omega \geqslant \frac{2 m_{e} c^{2}}{\hbar} \approx 2.47 \times 10^{20} \mathrm{~Hz}
$$

The central frequency of the (Planck) distribution of cosmic microwave background photons is 160GHz. The blueshift factor to transform a typical counter-directed CMB photon to the rest frame of the spacecraft is then 
$\omega=\gamma(1+v / c) \times 10^{12} \mathrm{~Hz}$, where $v$ is the velocity of the spacecraft and $\gamma=\left(1-v^{2} / c^{2}\right)^{-1 / 2}$. So the condition for pair creation in terms of spacecraft speed is:

$$
\gamma\left(1+\frac{v}{c}\right) \geqslant 2.47 \times 10^{8}
$$

corresponding to a $\gamma$ of about $\gamma \approx 1.24 \times 10^{8}$, or $v / c \approx 1-3.3 \times 10^{-17}$.

How much energy will be dissipated at this speed or above due to pair production? There are 412 cosmic microwave photons in the Universe per cubic centimeter (see Eq. (13) below). Assuming the spacecraft has a crosssectional area of $A$ (measured in $\mathrm{cm}^{2}$ ), it will be colliding with approximately $1.2 \times 10^{13} \mathrm{~A}$ microwave background photons per second. Each created electron-positron pair dissipates $1.6 \times 10^{-13}$ Joules of energy, thus the viscousdissipative effect of the cosmic background on the spacecraft would be energy dissipation of approximately $2 \mathrm{~A}$ Joules per second. Assuming an effective cross-sectional area of say 100 square meters, $A \approx 10^{6} \mathrm{~cm}^{2}$, the dissipative effect is about 2 million Joules per second, but with the time rate of change measured in the rest frame of the cosmic microwave background. In the spacecraft's rest frame, 1 "cosmic background second" corresponds to $1 / \gamma$ proper time seconds; so energy dissipation is a factor of $\gamma$ higher, or about $2.5 \times 10^{14}$ Joules per proper time second, which is a large drag to be overcome by the spacecraft engines just to keep it moving at constant velocity. Therefore shutting down the propulsion system when the desired speed is reached is not an option at large $\gamma$ values. In general, the drag on the spacecraft scales as $\gamma^{2}$, so, for example, at $\gamma \leqslant 10$ the drag will be less than a few Joules per second and thus negligible.

At the next level, we can imagine technology so advanced that the spacecraft "hull" does not contain any ordinary baryonic matter but some other kind of physical matter field. However, regardless of how advanced the spacecraft hull material can be, at sufficiently relativistic velocities pair production of CMB photons via the Schwinger breakdown of vacuum will become significant. The Schwinger limit corresponds to the electric field strength at which spontaneous electron-positron pair production from the electromagnetic field (photons) becomes energetically favorable:

$$
e E_{S} \frac{h}{m_{e} c}=2 m_{e} c^{2}
$$

or

$$
E_{S}=\frac{m_{e}{ }^{2} c^{3}}{\pi e \hbar} \approx 1.4 \times 10^{14}\left(\mathrm{erg} / \mathrm{cm}^{3}\right)^{1 / 2}
$$

Here $h / m_{e} c$ is the electron Compton wavelength, and $e$ is the electronic charge. Let $\delta$ be the effective skin depth for the interaction/penetration of high energy photons into the "material" making up the spacecraft (or its forward shield). Then the colliding gamma rays will pair-create provided the electric field $E$ associated with their localization at the length scale $\delta$ and with their frequency $\omega$ in the rest frame of the spacecraft exceeds the Schwinger limit:

$$
E \approx \sqrt{\frac{8 \pi \hbar \omega}{\delta^{3}}} \geqslant E_{S}=\frac{m_{e}{ }^{2} c^{3}}{\pi e \hbar}
$$

In terms of the frequency $\omega$ in the rest frame of the spacecraft, the condition for pair creation is

$$
\omega \geqslant \frac{m_{e}^{4} c^{6} \delta^{3}}{8 \pi^{3} e^{2} \hbar^{3}}=\frac{E_{S}^{2}}{8 \pi \hbar} \delta^{3} \approx 7.8 \times 10^{51} \mathrm{~Hz} \times[\delta(\mathrm{cm})]^{3}
$$

Again with the central $\mathrm{CMB}$ frequency of $160 \mathrm{GHz}$, the blueshift factor to transform the $\mathrm{CMB}$ photon to the rest frame of the spacecraft is $\omega=\gamma(1+v / c) \times 10^{12} \mathrm{~Hz}$ as before. So the condition for nucleus-mediated pair creation in terms of spacecraft speed and $\delta$ is:

$$
\gamma\left(1+\frac{v}{c}\right) \geqslant 7.8 \times 10^{39}[\delta(\mathrm{cm})]^{3}
$$

Let us assume the skin-depth $\delta$ for high frequency radiation is on the order of a micron: $\delta=10^{-4} \mathrm{~cm}$. This gives

$$
\gamma\left(1+\frac{v}{c}\right) \geqslant 7.8 \times 10^{27}
$$


corresponding to a $\gamma$ of about $\gamma \approx 4 \times 10^{27}$, or $v / c \approx 1-3 \times 10^{-56}$.

Even if the speed of spacecraft is below the limits required for pair production, simply scattering the microwave photons creates drag. How big is that drag? To calculate this in order of magnitude, assume each photon is scattered by reflecting directly back as it hits the spacecraft. This corresponds to a momentum transfer to each photon of about

$$
2 \frac{\hbar \omega}{c}=\gamma\left(1+\frac{v}{c}\right) 6.6 \times 10^{-26} \mathrm{~g} \mathrm{~cm} / \mathrm{sec}=\gamma\left(1+\frac{v}{c}\right) 6.6 \times 10^{-31} \mathrm{Nt} \mathrm{sec}
$$

(as measured in the rest frame of the spacecraft). Taking into account all photons scattering off the frontal cross section per unit (spacecraft) proper time, we obtain a viscous drag force on the spacecraft of about

$$
A\left(\mathrm{~cm}^{2}\right) \gamma^{2}\left(1+\frac{v}{c}\right) 8 \times 10^{-18} \mathrm{Nt}
$$

This will become a significant obstacle at speeds for which $\gamma \geqslant 10^{8}$ or $v / c \gtrsim 1-5 \times 10^{-17}$, which is just below the velocity threshold for nucleus-mediated pair production.

We summarize various effects caused by interactions with matter and CMB photons in the table below:

\begin{tabular}{|c|c|l|}
$\gamma$ & Speed & \multicolumn{1}{|c|}{ Effect } \\
\hline 1.3 & $.69 c$ & pion production in nucleon-nucleon collisions \\
\hline 9.1 & $.994 c$ & anti-proton production in nucleon-nucleon collisions \\
\hline $10^{8}$ & $\left(1-5 \times 10^{-17}\right) c$ & nucleus-mediated pair production in collisions with CMB photons \\
\hline $4 \times 10^{27}$ & $\left(1-3 \times 10^{-56}\right) c$ & pair production in collisions with CMB photons via Schwinger vacuum breakdown \\
\hline
\end{tabular}

\section{Lorentz-transformed Appearance of CMB}

We now turn to examine how the CMB photon distribution would appear to an observer in the rest frame of the spacecraft. In the rest frame of the $\mathrm{CMB}$, the photon distribution is thermal with the Planck density

$$
n(\vec{k}) d^{3} k=\frac{1}{4 \pi^{3}} \frac{1}{e^{\hbar \omega / k_{B} T}-1} d^{3} k
$$

where $\omega=c|\vec{k}|, k_{B}$ is Boltzmann's constant, and the left hand side denotes the number of photons per unit volume with wave vectors lying in a cell of size $d^{3} k$ centered around $\vec{k}$. From Eq. (11) we can deduce, among other things, that the number of photons per unit volume with frequencies between $\omega$ and $\omega+d \omega$ is

$$
n(\omega) d \omega=\frac{1}{\pi^{2} c^{3}} \frac{1}{e^{\hbar \omega / k_{B} T}-1} \omega^{2} d \omega
$$

Equation (12) is the more familiar form of the Planck (Bose-Einstein) distribution giving rise to, for example, the total number of photons per unit volume

$$
\int_{0}^{\infty} n(\omega) d \omega=\frac{2 k_{B}{ }^{3} T^{3}}{\pi^{2} c^{3} \hbar^{3}} \zeta(3) \approx 412 \text { photons } / \mathrm{cm}^{3}
$$

where $T \approx 2.73^{\circ} \mathrm{K}$ for the $\mathrm{CMB}$, and $\zeta$ is the Riemann zeta function.

Consider now a CMB photon with 4-momentum vector

$$
p=\hbar \omega\left(\partial_{t}+n_{x} \partial_{x}+n_{y} \partial_{y}+n_{z} \partial_{z}\right)
$$

in the rest frame of the CMB spanned by the orthonormal basis $\left(\partial_{t}, \partial_{x}, \partial_{y}, \partial_{z}\right)$. We will use units in which $c=1$ unless we explicitly switch back to standard units for physical interpretation, so here $\vec{n}$ is a unit 3-vector in the direction of 
the photon's propagation. The rest frame of the spacecraft is spanned by the basis vectors

$$
\begin{aligned}
& e_{0}=\gamma\left(\partial_{t}+v \partial_{x}\right) \\
& e_{1}=\gamma\left(v \partial_{t}+\partial_{x}\right) \\
& e_{2}=\partial_{y} \\
& e_{3}=\partial_{z}
\end{aligned}
$$

The same photon is seen in the rest frame of the spacecraft as having the 4-momentum

$$
p=\hbar \omega \gamma\left(1-v n_{x}\right)\left[e_{0}+\frac{n_{x}-v}{1-v n_{x}} e_{1}+\frac{n_{y}}{\gamma\left(1-v n_{x}\right)} e_{2}+\frac{n_{z}}{\gamma\left(1-v n_{x}\right)} e_{3}\right]
$$

which is the 4-vector Eq. (14) acted on by the Lorentz transformation matrix

$$
\Lambda=\left[\begin{array}{cccc}
\gamma & -\gamma v & 0 & 0 \\
-\gamma v & \gamma & 0 & 0 \\
0 & 0 & 1 & 0 \\
0 & 0 & 0 & 1
\end{array}\right]
$$

Similarly, a photon with 4-momentum vector

$$
p=\hbar \omega\left(e_{0}+m_{x} e_{1}+m_{y} e_{2}+m_{z} e_{3}\right)
$$

in the rest frame of the spacecraft is seen as having 4-momentum

$$
p=\hbar \omega \gamma\left(1+v m_{x}\right)\left[\partial_{t}+\frac{m_{x}+v}{1+v m_{x}} \partial_{x}+\frac{m_{y}}{\gamma\left(1+v m_{x}\right)} \partial_{y}+\frac{m_{z}}{\gamma\left(1+v m_{x}\right)} \partial_{z}\right]
$$

in the rest frame of the $\mathrm{CMB}$, which is just the 4-vector Eq. (18) acted on by the inverse of $\Lambda$

$$
\Lambda^{-1}=\left[\begin{array}{cccc}
\gamma & \gamma v & 0 & 0 \\
\gamma v & \gamma & 0 & 0 \\
0 & 0 & 1 & 0 \\
0 & 0 & 0 & 1
\end{array}\right]
$$

The distribution function for photon density transforms under a coordinate change according to the general identity

$$
\hat{n}\left(\vec{k}^{\prime}\right)=n\left[\vec{k}\left(\vec{k}^{\prime}\right)\right]\left|\frac{\partial \vec{k}}{\partial \overrightarrow{k^{\prime}}}\right|
$$

where $\hat{n}$ is the density in the Lorentz transformed frame, and the right hand side involves the coordinates $\left\{k_{x}, k_{y}, k_{z}\right\}$ expressed as functions of the transformed coordinates $\left\{k_{x}^{\prime}, k_{y}^{\prime}, k_{z}^{\prime}\right\}$ where the last term is the Jacobian determinant. According to Eq. (19), we have

$$
\vec{k}\left(\vec{k}^{\prime}\right)=\left[\gamma\left(k_{x}^{\prime}+v \omega^{\prime}\right), k_{y}^{\prime}, k_{z}^{\prime}\right]
$$

and

$$
\omega^{2}=k_{x}^{2}+k_{y}^{2}+k_{z}^{2}=\gamma^{2}\left(\omega^{\prime}+v k_{x}^{\prime}\right)^{2}
$$



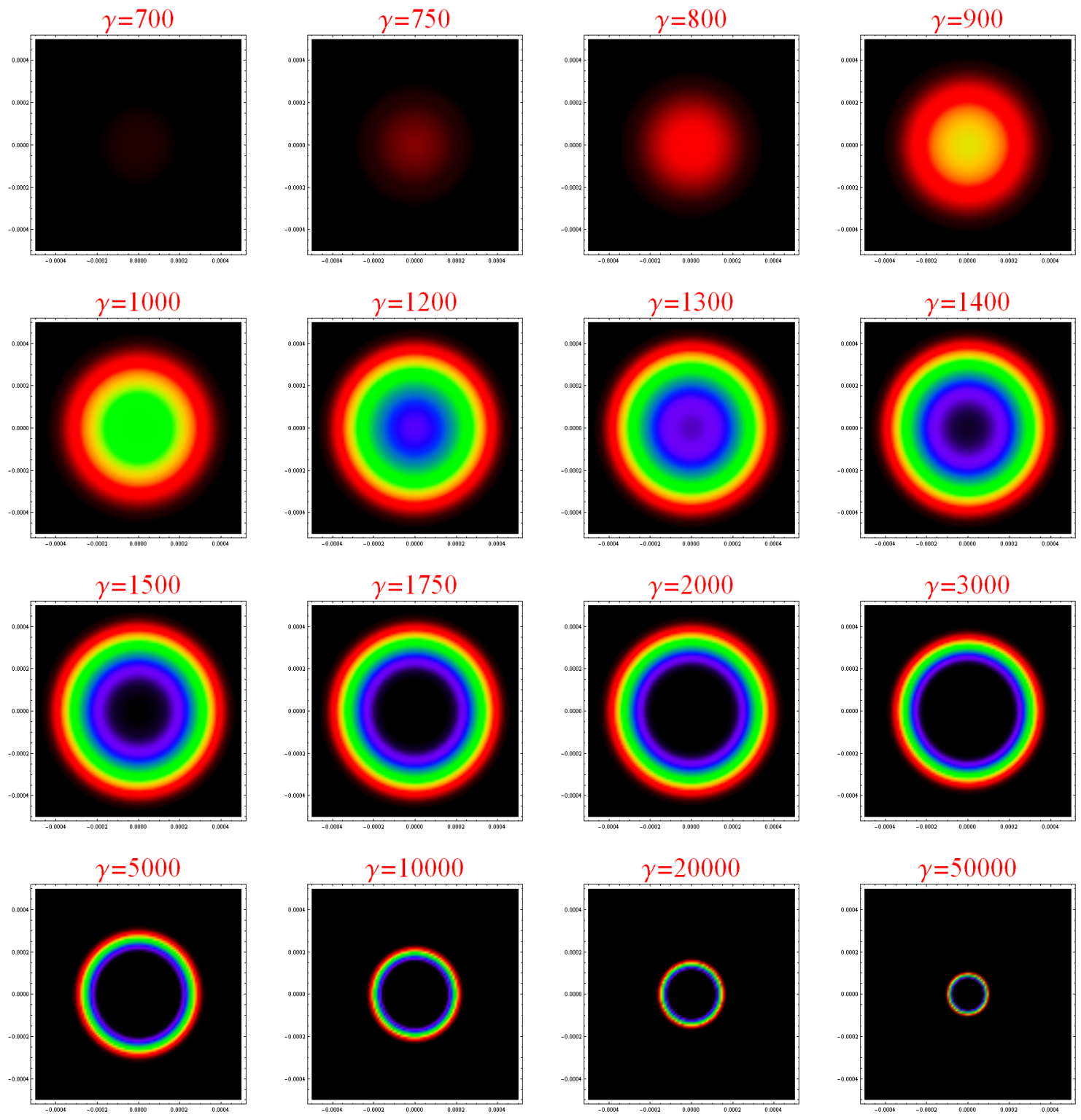

FIG. 1: How the cosmic microwave background would appear to a forward facing observer located in a relativistic spacecraft. The plots are for increasing spacecraft speeds (relative to the $\mathrm{CMB}$ ) as measured by the Lorentz factor $\gamma$. The appearance is approximately the same as that of a thermal distribution with latitudinally-varying temperature. If we use Wien's displacement law, the apparent color of the CMB is the wavelength at which the spectral radiance of the Planck distribution reaches its peak. The visible colors form concentric rings confined to a narrow cone in the polar angle $\theta$, with the zenith $\theta=0$ fixed along the forward $x$-direction. The plots depict the apparent "celestial sky" parameterized with the $y, z$ coordinates of a unit sphere whose north pole lies on the $x$-axis. The first light from blue-shifted CMB photons is visible in red at around $\gamma=700$. For an explanation of the variation in shape and size of the color rings with increasing $\gamma$ see the discussion following Eq. (27).

The Jacobian determinant is easily computed:

$$
\left|\frac{\partial \vec{k}}{\partial \vec{k}^{\prime}}\right|=\gamma\left(1+\frac{v k_{x}^{\prime}}{\omega^{\prime}}\right)
$$

Combining Eqs. (21)-(24) with Eq. (11) and dropping the primes on transformed quantities for notational conve- 
nience, we obtain the $\mathrm{CMB}$ photon distribution as seen in the rest frame of the spacecraft

$$
\hat{n}(\vec{k})=\frac{1}{4 \pi^{3}} \frac{\gamma\left(1+\frac{v k_{x}}{\omega}\right)}{e^{\hbar \gamma\left(\omega+v k_{x}\right) / k_{B} T}-1}
$$

where $\omega=c \sqrt{k_{x}^{2}+k_{y}^{2}+k_{z}^{2}}$.

It is an interesting exercise to plot the appearance of the CMB to a forward facing observer located in the spacecraft (Fig. 1). For qualitative description, we can neglect the Jacobian factor in the numerator of Eq. (25), and notice that for photons moving opposite to the "look" direction the wave vector obeys $k_{x}=-(\omega / c) \cos \theta$, where $\theta$ measures the angle between the look direction and the forward $(x-)$ direction. Thus to the forward-facing observer the CMB appears to have a thermal distribution with latitudinally-varying temperature

$$
T(\theta)=\frac{T}{\gamma\left(1-\frac{v}{c} \cos \theta\right)}=T \frac{\sqrt{1-\frac{v^{2}}{c^{2}}}}{\left(1-\frac{v}{c} \cos \theta\right)}
$$

If we use Wien's displacement law and approximate the apparent color of the CMB with the wavelength at which the spectral radiance of the Planck distribution reaches its peak, then the color visible at polar angle $\theta$ is given by the wavelength

$$
\lambda(\theta)=\frac{b}{T(\theta)}=\frac{b}{T} \frac{\left(1-\frac{v}{c} \cos \theta\right)}{\sqrt{1-\frac{v^{2}}{c^{2}}}}=\frac{b}{T}\left(\gamma-\sqrt{\gamma^{2}-1} \cos \theta\right)
$$

where $b$ is Wien's displacement constant equal to $2.9 \times 10^{-3}{ }^{\circ} \mathrm{K} \mathrm{m}$. As shown in Fig.1, the visible frequencies are confined to a narrow cone in $\theta$ around the forward direction, and the constant color temperatures form rings around $\theta=0$ whose shape and size vary with increasing $\gamma$. This is closely related to the phenomeneon of (relativistic) aberration, which describes how the appearance of an optical source varies in a geometric, frequency-independent way under a Lorentz transformation [5]. To understand this variation better, it may help to examine the angle $\theta$ at which the relativistic Doppler shift of the incoming $\mathrm{CMB}$ photon is zero (i.e., no shift in frequency). The condition for this to happen is

$$
\gamma\left(1-\frac{v}{c} \cos \theta\right)=\gamma-\sqrt{\gamma^{2}-1} \cos \theta=1
$$

The solution $\theta_{0}$ to Eq. (28) is

$$
\theta_{0}=\arccos \left(\frac{\gamma-1}{\sqrt{\gamma^{2}-1}}\right)=\arccos \left(\sqrt{\frac{\gamma-1}{\gamma+1}}\right)
$$

The CMB photons appear blue-shifted to our forward-facing observer for angles $\theta$ less than $\theta_{0}$ and red-shifted for angles $\theta \geqslant \theta_{0}$. For small velocities ( $\gamma$ close to 1$), \theta_{0}$ is near $\pi / 2$ as would be expected. However, just as in relativistic aberration, in the limit of large velocities (large $\gamma$ ) $\theta_{0}$ approaches zero:

$$
\theta_{0}=\sqrt{\frac{2}{\gamma}}-\frac{1}{3 \sqrt{2} \gamma^{3 / 2}}+O\left(\frac{1}{\gamma^{5 / 2}}\right)
$$

which explains the progressive narrowing at very large $\gamma$ values of the visible ring in Fig. 1.

\section{Signature of CMB Scattered from a Relativistic Spacecraft}

The possibility of detecting radiation associated with distant relativistic spacecraft has been discussed in the literature before [6-8]. These discussions mostly focus on detecting radiation from spacecraft engines or light from nearby stars reflecting off the spacecraft. Our approach is different in that we do not speculate on possible propulsion technologies but are interested in how a large relativistic object would interact with the interstellar/intergalactic medium 
and mainly with the $\mathrm{CMB}$ radiation. As a baryonic spacecraft travels at relativistic speeds it will interact with the $\mathrm{CMB}$ through scattering to cause a frequency shift that could be detectable on Earth with current technology.

Now we consider the characteristic signature of this reflected CMB radiation; specifically, CMB photons reflected by a surface area element of area $A$ moving with velocity $v$ in the $x$ direction. Let the normal to the surface element $A$ be described by a unit 3-vector $\hat{s}$ in the spacecraft restframe. Then every photon impacting $A$ is reflected back according to

$$
\vec{k} \longrightarrow \vec{k}-2(\vec{k} \cdot \hat{s}) \hat{s}
$$

From a far-field perspective the area element $A$ would appear as a point source. We set up a coordinate system in the CMB rest frame where $A$ is at $\vec{x}=\vec{v} t=v t \hat{x}$ and the observation point is at $\vec{r}$. Notice that the time coordinate is chosen in such a way that the spacecraft's worldline passes through the coordinate origin at time $t=0$. The radiation detected at $\vec{r}$ at time $t$ would have been emitted from the spacecraft at the retarded time $\sigma$ which is the time at the intersection between the past null cone of the event $(\vec{r}, t)$ and the worldline of the spacecraft. Hence the equation for $\sigma$ is

$$
c(t-\sigma)=\sqrt{(x-v \sigma)^{2}+y^{2}+z^{2}}
$$

The solution to Eq. (32) is

$$
\sigma_{\left(\begin{array}{l}
\text { ret } \\
\text { adv }
\end{array}\right)}=\gamma^{2}\left(t-\frac{v x}{c^{2}} \mp \frac{1}{c \gamma} \sqrt{\gamma^{2}(x-v t)^{2}+y^{2}+z^{2}}\right)
$$

where the retarded (advanced) solution represents intersection with the past (future) null cone of the observation point $(\vec{r}, t)$. We will only use the retarded solution

$$
\sigma=\sigma(\vec{r}, t) \equiv \sigma^{(\mathrm{ret})}(\vec{r}, t)=\gamma^{2}\left(t-\frac{v x}{c^{2}}-\frac{1}{c \gamma} \sqrt{\gamma^{2}(x-v t)^{2}+y^{2}+z^{2}}\right)
$$

Only reflected photons with wave vectors parallel to $(\vec{r}-\vec{v} \sigma)$ will be detected. There are two Lorentz transformations involved: First to find the photon distribution in the spacecraft rest frame according to Eq. (25), and second to transform the distribution reflected according to Eq. (31) back into the CMB rest frame, which is accomplished by the same formulas as in Eqs. (16)-(25). According to Eqs. (16)-(25) and Eq. (31), the spectral intensity of the reflected radiation in the $\mathrm{CMB}$ rest frame at position $\vec{r}$ and at time $t$ would be (measured in number of photons of frequency $\omega$ per unit area per unit time per unit frequency)

$$
I(\vec{r}, t, \omega)=\frac{c A}{4 \pi|\vec{r}-\vec{v} \sigma|^{2}} \frac{\omega^{2}}{\pi^{2} c^{3}} \frac{\gamma^{2}\left[1+\left(\frac{\vec{v}}{c|\vec{r}-\vec{v} \sigma|} \cdot(\vec{r}-\vec{v} \sigma-2[(\vec{r}-\vec{v} \sigma) \cdot \hat{s}] \hat{s})\right]^{2}\right.}{\exp \left[\frac{\gamma^{2} \hbar \omega}{k_{B} T}\left[1+\left(\frac{\vec{v}}{c|\vec{r}-\vec{v} \sigma|} \cdot(\vec{r}-\vec{v} \sigma-2[(\vec{r}-\vec{v} \sigma) \cdot \hat{s}] \hat{s})\right]^{2}\right]-1\right.}
$$

where $\sigma$ is given by Eq. (34) and $\vec{v}=v \hat{x}$. For a general reflecting surface $S$ made up of many small surface elements, the total intensity can be computed as a surface integral of Eq. (35)

$$
I_{T}(\vec{r}, t, \omega)=\frac{\omega^{2}}{4 \pi^{3} c^{2}} \oint_{S} \frac{1}{|\vec{r}-\vec{v} \sigma|^{2}} \frac{\gamma^{2}\left[1+\left(\frac{\vec{v}}{c|\vec{r}-\vec{v} \sigma|} \cdot(\vec{r}-\vec{v} \sigma-2[(\vec{r}-\vec{v} \sigma) \cdot \hat{s}] \hat{s})\right]^{2}\right.}{\exp \left[\frac{\gamma^{2} \hbar \omega}{k_{B} T}\left[1+\left(\frac{\vec{v}}{c|\vec{r}-\vec{v} \sigma|} \cdot(\vec{r}-\vec{v} \sigma-2[(\vec{r}-\vec{v} \sigma) \cdot \hat{s}] \hat{s})\right]^{2}\right]-1\right.} d A
$$

We will limit our attention in this paper to reflection from a single surface element $A$ as given in Eq. (35). Using Wien's displacement law, the peak frequency of the reflected radiation Eq. (35) is given by

$$
\nu_{\max }(\vec{r}, t)=\frac{c}{b \gamma^{2}} \frac{T}{\left[1+\left(\frac{\vec{v}}{c|\vec{r}-\vec{v} \sigma|} \cdot(\vec{r}-\vec{v} \sigma-2[(\vec{r}-\vec{v} \sigma) \cdot \hat{s}] \hat{s})\right]^{2}\right.}
$$


corresponding to a Planck distribution with effective temperature

$$
T_{\text {eff }}(\vec{r}, t)=\frac{T}{\gamma^{2}\left[1+\left(\frac{\vec{v}}{c|\vec{r}-\vec{v} \sigma|} \cdot(\vec{r}-\vec{v} \sigma-2[(\vec{r}-\vec{v} \sigma) \cdot \hat{s}] \hat{s})\right]^{2}\right.}
$$

On the other hand, the relative intensity profile is determined by the dimensionless multiplicative factor

$$
i(\vec{r}, t, \omega)=\frac{A}{|\vec{r}-\vec{v} \sigma|^{2}} \gamma^{2}\left[1+\left(\frac{\vec{v}}{c|\vec{r}-\vec{v} \sigma|} \cdot(\vec{r}-\vec{v} \sigma-2[(\vec{r}-\vec{v} \sigma) \cdot \hat{s}] \hat{s})\right]^{2}\right.
$$
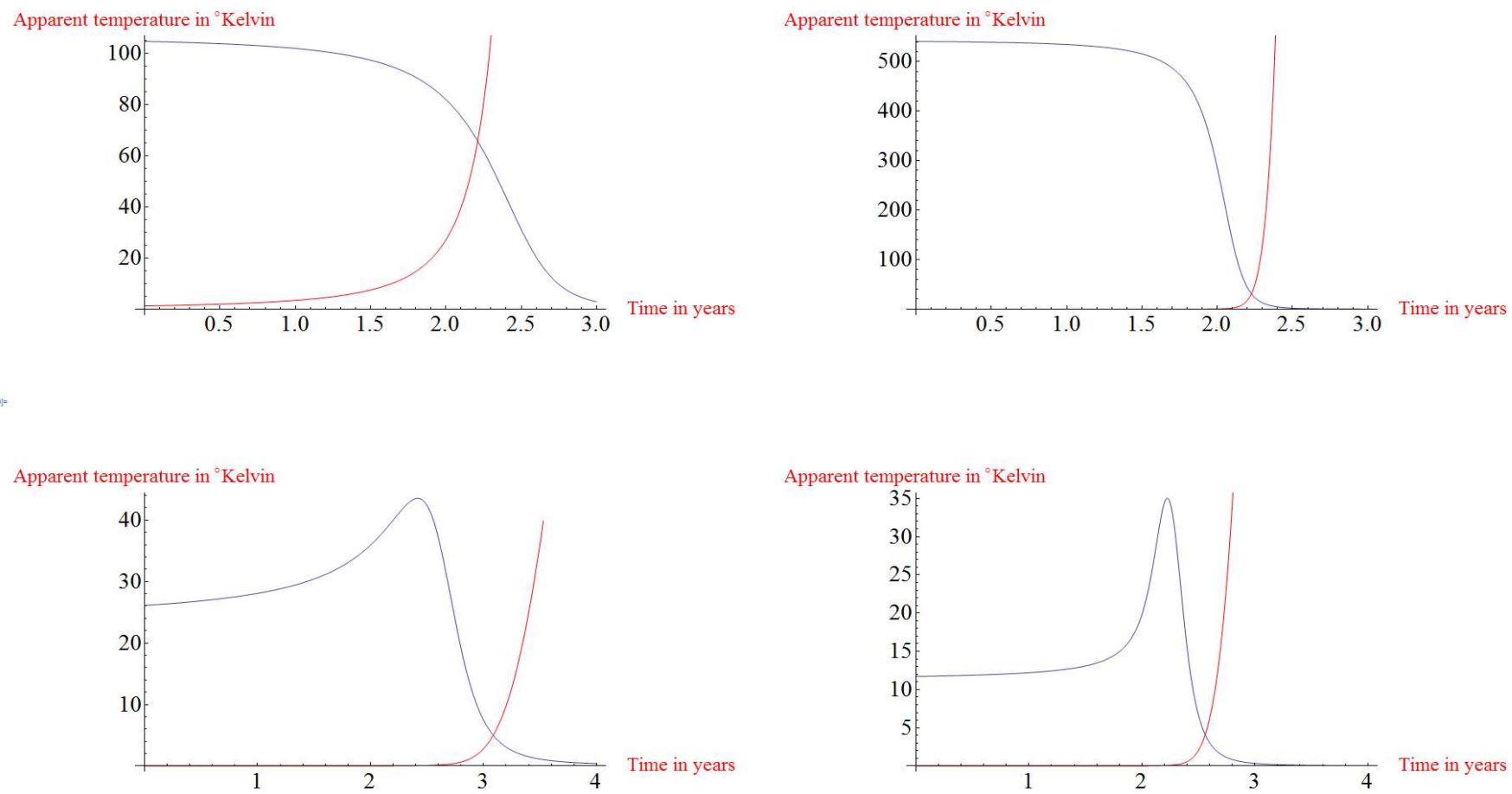

FIG. 2: Some examples of signal signatures of CMB scattering from relativistic spacecraft. In all plots the observation point is located at $x=10$ lightyears, $y=z=1$ lightyear. The time axis origin is at $t=8$ years (spacecraft is moving along the $x$-direction and is at the coordinate origin at time $t=0$ ). In the upper row, we plot the apparent (effective) temperature of the signal from a surface element orthogonal to the $x$-direction (the direction of motion) for spacecraft velocities $v=0.95 c$ and $v=0.99 c$, respectively. In the lower row, the plots are for a surface element tilted $10^{\circ}$ from orthogonal to the $x$-direction but still parallel to the $z$-direction, with the same two velocities. The red curves are plots of the multiplicative factor Eq. (39) in arbitrary units, showing the relative intensity profile of the received radiation as a function of time. The decrease in temperature with increasing $\gamma$ in the second row of plots might seem counter-intuitive at first; but it is due to the same phenomenon of relativistic aberration discussed above [cf. Eq. (30)]; namely, the $10^{\circ}$ tilt in the reflecting surface is enough to put the reflected beam outside the cone of blueshift for the $\gamma$ value corresponding to $v=0.99 \mathrm{c}$.

Some examples of signal signatures resulting from Eqs. (38)-(39) are shown in Figs. 2 and 3. The salient features of the signal are a rapid drop in temperature accompanied by a rapid rise in intensity, along with the motion of the 
source with respect to a reference frame fixed to distant quasars, which should be observable. No natural source of $\mathrm{THz}$ to infrared radiation in the known Universe is likely to have time variability of the kind depicted in Figs. 2-3. Note that increasing velocity (increasing $\gamma$ ) manifests itself in a steepening of the cooling curve, along with an overall increase in temperature for a surface element oriented orthogonal to the direction of motion.
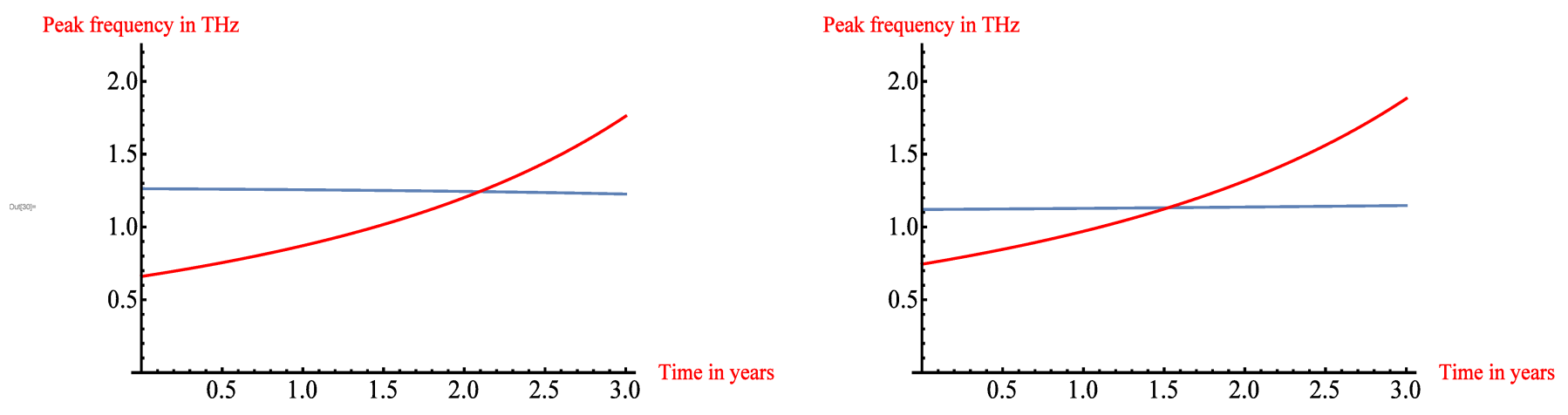

FIG. 3: A further example of signal signature for $\mathrm{CMB}$ scattering from relativistic spacecraft. The spacecraft speed in this case is a relatively small $v=0.64 c(\gamma=1.3)$. As in Fig. 2, the observation point is located at $x=10$ lightyears, $y=z=1$ lightyear. The time axis origin is at $t=8$ years (spacecraft is moving along the $x$-direction and is at the coordinate origin at time $t=0$ ). This time we plot the peak frequency of the signal; on the left from a surface element orthogonal to the $x$-direction, and on the right from a surface element tilted $10^{\circ}$ from that orthogonal but still parallel to the $z$-direction. The red curve is again a plot of the multiplicative factor Eq. (39) in arbitrary units, showing the relative intensity profile of the received radiation as a function of time. The salient features recognizable in Fig. 2 are much less prominent, owing to the comparatively small value of $\gamma$ in this case.

Finally, we note the similarity of CMB scattering from macroscopic relativistic objects to the Sunyaev-Zeldovich (S-Z) effect in cosmology. The S-Z effect is the spectral distortion of the CMB caused by inverse Compton scattering of microwave background photons from energetic intracluster thermal electrons (intracluster referring to clusters of galaxies) [9, 10]. S-Z scattering has been observed [11, 12]. The intracluster electrons have temperatures of order 3 to $15 \mathrm{keV}$, which correspond to gamma factors of $\gamma \approx 1.01-1.03$, which correspond to thermal velocities of $v / c \approx 0.15-0.25$. Therefore, while these electrons are relativistic, they are far from the ultra-relativistic speeds we are interested in. However, the observed S-Z effect in cosmology is qualitatively similar to what we discuss above.

On the other hand, merger shocks in the collisions of clusters of galaxies can produce non-thermal electrons at energies from $300 \mathrm{MeV}(\gamma \approx 300)$ up to $10^{6} \mathrm{MeV}\left(\gamma \approx 2 \times 10^{6}\right)$, so these electrons are more relevant to our discussion [13]. In fact, these ultra-relativistic electrons, protons, and heavier ions accelerated by merger shocks loose energy via inverse Compton, pair production, and other effects mediated by collisions with the cosmic microwave photons, much as we discussed for the ultrarelativistic spaceship example. The S-Z effect in this case, which is exactly the same as the spaceship example except the relativistic object is a single particle, should be similar provided it is observable.

\section{Summary}

We have explored the physical interaction of a relativistic spacecraft with the interstellar and intergalactic mediums. Our main discussion focused on the interaction with the CMB where very little information currently exists and seems to have been overlooked. Central to our discussion was not how to obtain relativistic speeds but the consequences of traveling that fast. We discussed the interaction with baryonic matter in terms of high speed collisions to present a complete picture. In general one can imagine the same interactions that occur in a particle accelerator to 
occur between relativistic spacecraft and interstellar matter. Our assumption that matter-matter interactions can be dealt with when civilization can build relativistic spacecraft may prove false and may be a barrier that will prevent space travel with a large $\gamma$.

We looked at two special reference frames, the spacecraft frame, and the rest frame of the CMB to understand how the $\mathrm{CMB}$ is distorted or acquires an aberration from those view points. The scattering of the CMB from a relativistic spacecraft is very similar to the Sunyaev-Zedovich effect where the inverse Compton scattering instead occurs at a macroscopic level. Our calculation for what an observer on earth could detect predicts a very unusual signature that is unlikely to be caused by any naturally occurring object in the known universe. This result is independent of propulsion technology, but the ability to detect the signal from Earth depends on available detector technologies. We are currently working to predict how far can we see this signature given our current capability.

[1] P. A. Tipler \& R. A. Llewellyn, Modern Physics. W. H. Freeman and Co., New York, Fifth Edition (2008).

[2] S. Weinberg, Gravitation and Cosmology. Wiley, New York (1972).

[3] D. J. Griffiths, Introduction to Elementary Particles. John Wiley and Sons (1987).

[4] D. H. Perkins, Introduction to High Energy Physics. Addison-Wesley Press (1989).

[5] G. D. Scott and H. J. van Driel, Am. J. Phys. 38, 971-977 (1970).

[6] R. Zubrin, Detection of Extraterrestrial Civilizations via the Spectral Signature of Advanced Interstellar Spacecraft, in Progress in the Search for Extraterrestrial Life, ASP Conference Series, 74, 487 (1995).

[7] D. R. J. Viewing, C. Horswell, E. W. Palmer, JBIS 30, 99-104 (1977).

[8] J. C. Garcia-Escarfin \& P. Chamorro-Posada, arXiv. 1203: 3980 (2012).

[9] R. A. Sunyaev \& Y. B. Zeldovich, Comments Astrophys. Space Phys. 2, 66 (1970).

[10] R. A. Sunyaev \& Y. B. Zeldovich, Comments Astrophys. Space Phys. 4, 173 (1972).

[11] M. Birkinshaw, Phys. Reports 310, 97-196 (1999).

[12] J. E. Carlstrom, M. Joy, L. Grego, Astrophys. Journal 456, L75-L78 (1996).

[13] M. Markevitch \& A. Vikhlinin, Phys. Reports 443, 1 (2007). 